\title{
Iterative Strategies for Aftershock Classification in Automatic Seismic Processing Pipelines
}

\author{
Steven J. Gibbons ${ }^{1}$, Tormod Kværna ${ }^{1}$, David B. Harris ${ }^{2}$ and Douglas A. Dodge ${ }^{3}$ \\ ABSTRACT
}

Aftershock sequences following very large earthquakes present enormous challenges to nearrealtime generation of seismic bulletins. The increase in analyst resources needed to relocate an inflated number of events is compounded by failures of phase association algorithms and a significant deterioration in the quality of underlying fully automatic event bulletins. Current processing pipelines were designed a generation ago and, due to computational limitations of the time, are usually limited to single passes over the raw data. With current processing capability, multiple passes over the data are feasible. Processing the raw data at each station currently generates parametric datastreams which are then scanned by a phase association algorithm to form event hypotheses. We consider the scenario where a large earthquake has occurred and propose to define a region of likely aftershock activity in which events are detected and accurately located using a separate specially targeted semi-automatic process. This effort may focus on so-called pattern detectors, but here we demonstrate a more general grid search algorithm which may cover wider source regions without requiring waveform similarity. Given many well-located aftershocks within our source region, we may remove all associated phases from the original detection lists prior to a new iteration of the phase association algorithm. We provide a proof-of-concept example for the 2015 Gorkha sequence, Nepal, recorded on seismic arrays of the International Monitoring System. Even with very conservative conditions for defining event hypotheses within the aftershock source region, we can automatically remove about half of the original detections which could have been generated by Nepal earthquakes and reduce the likelihood of false associations and spurious event

\footnotetext{
${ }^{1}$ NORSAR, Kjeller, Norway.

${ }^{2}$ Deschutes Signal Processing LLC, Maupin, Oregon, USA.

${ }^{3}$ Lawrence Livermore National Laboratory, Livermore, California, USA.
} 
hypotheses. Further reductions in the number of detections in the parametric data streams are likely using correlation and subspace detectors and/or empirical matched field processing. 


\section{INTRODUCTION}

Many seismological data centers around the world aim to provide the most accurate and rapid characterization of seismicity possible. In most cases, these focus on specific regions and provide an information service for mitigating the impacts of earthquakes (e.g. the Pacific Northwest Seismic Network). However, some data centers have global scope. The National Earthquake Information Center (NEIC) in Golden, Colorado, for example aims to provide information on all earthquakes above magnitude 3.0 in the United States and 5.0 globally. The verification regime for the Comprehensive Nuclear-Test-Ban Treaty (CTBT) includes both a global seismic network (part of the International Monitoring System or IMS) and the International Data Center (IDC) in Vienna which collects and processes IMS data to generate a Reviewed Event Bulletin (REB) for nation states. The nuclear explosion monitoring missions of some nations may also include an independent National Technical Means to detect and locate seismic events globally, in addition to the services provided by the IDC.

The manually reviewed products generated by such data centers are based on the output from automatic procedures. The current basis for automatic event bulletin generation at the IDC is called Global Association or GA (Le Bras et al., 1994) and extends the principles of Generalized Beamforming or GBF (Ringdal and Kværna, 1989) and the Intelligent Monitoring System of Bache et al. (1993). The concepts of the existing processing pipelines at the IDC are illustrated in Figure 1. The datastreams (panel a) are present for many stations in the global network, which may be either 3component or array stations. Arrays have been fundamental in the seismic monitoring of nuclear explosions (e.g. Douglas, 2013) and this was very much the case when the IMS and IDC were designed (e.g. Dahlman et al. 2009, 2011). While only one stream is displayed here for each station, in practice many different detection traces are formed for each station. For 3-component stations, detection processes can be run independently on different rotations of the orthogonal components to seek P- and S- phases from different directions, and processed in multiple frequency bands in order to optimize the signal-to-noise ratio (SNR) for a broad range of anticipated signals. For seismic arrays, the situation is even more complicated with an extensive beam deployment steering 
numerous beams with carefully selected combinations of parameters for optimizing the detection of signals from all anticipated seismic sources. For all stations, a detection reduction and parameter estimation process will result in parametric datastreams (Figure $1 \mathrm{~b}$ ) whereby, for a given trigger time, we have a set of parameters describing the nature of the detection. These parameters include typically SNR, apparent velocity, backazimuth, frequency content, and amplitude. A GA-type algorithm will determine a set of event hypotheses which best explain the sets of parametric data, i.e. an automatic event bulletin (Figure $1 \mathrm{c}$ ). Four events have been formed from the parametric data streams in this illustrative example; three of these come from essentially the same geographical location but are placed at some distance from each other due to deficiencies in the various automatic stages of the process. The analyst then compiles a reviewed event bulletin (Figure 1d) based upon revisions of events found in the automatic bulletin(s). With access to the waveform data (e), and signal processing tools, event definitions can be modified until deemed satisfactory by the analyst. Event hypotheses that are clearly false are deleted and new events that were not identified by the automatic processing are added if the analyst sees sufficient evidence. 
a)

Waveform datastreams

b)

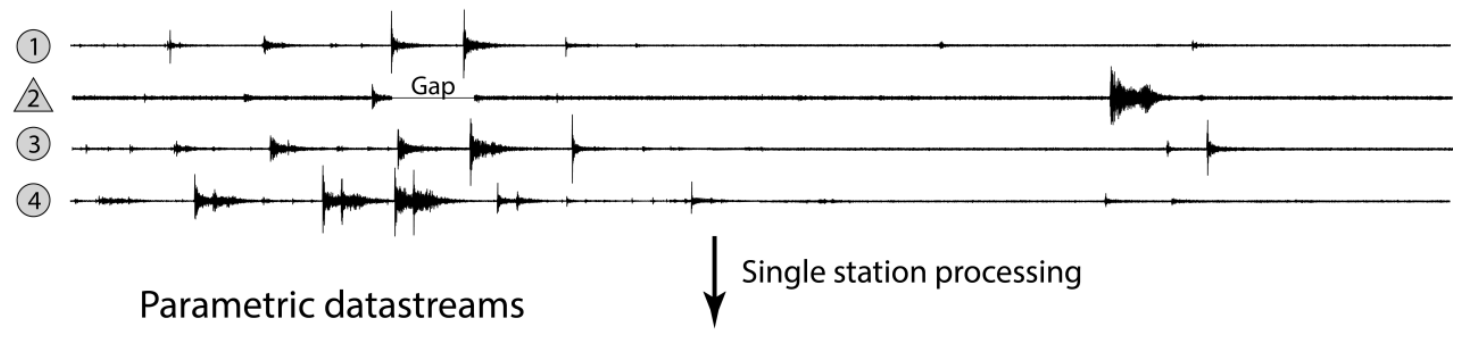

c)
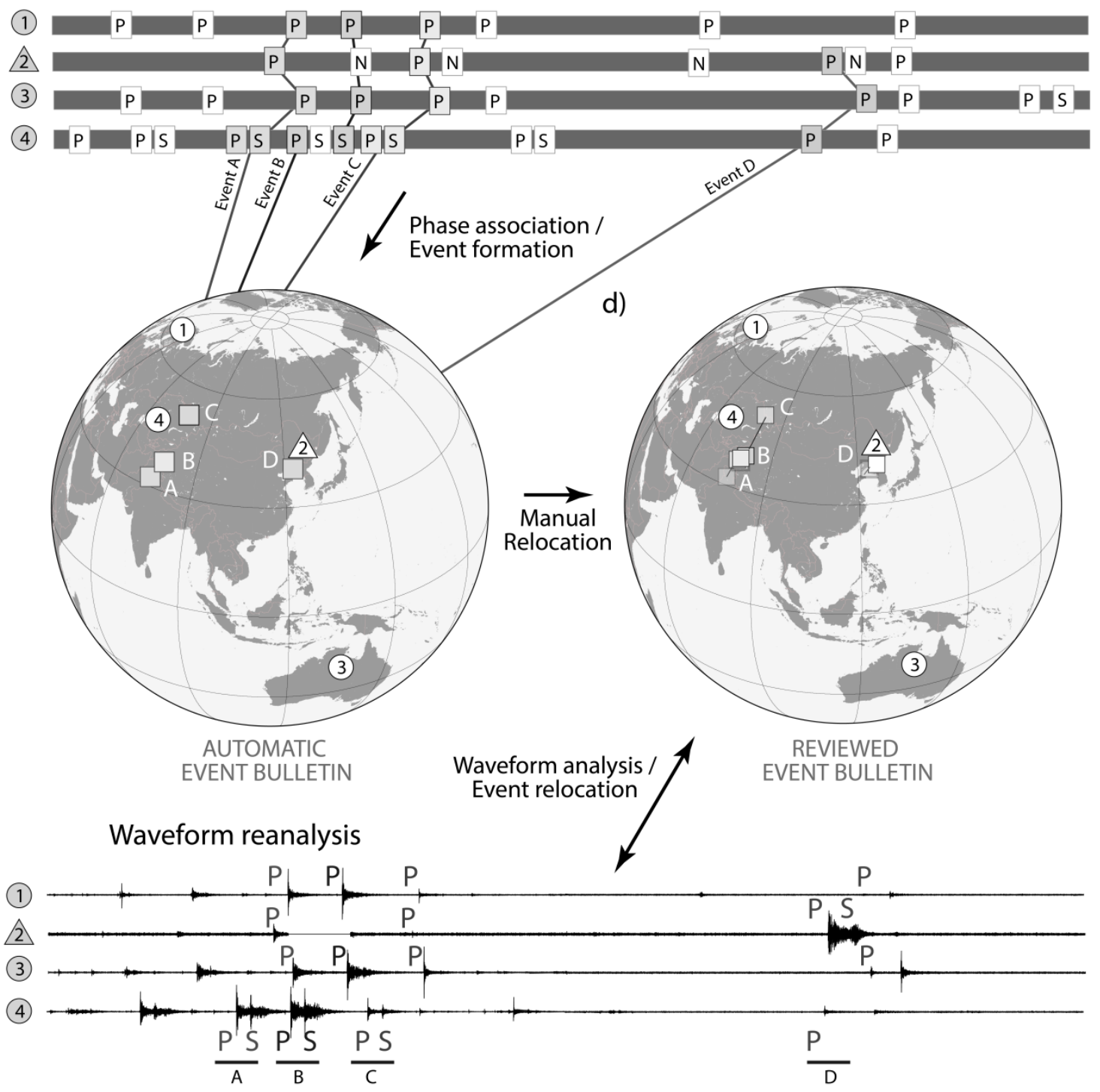

Figure 1 The classical processing pipeline displayed schematically for a global network. Waveforms

from arrays and 3-C stations are processed (a) and converted to parametric detection lists (b). A

global phase association algorithm then attempts to construct event hypotheses from these

detections (c) creating an automatic event bulletin which is then reviewed manually (d). The

analyst review includes reassessment of the waveform data (e). 
The processes in panels (a), (b) and (c) of Figure 1 all run fully automatically with a minimum of human supervision; the labor-intensive part of the process is the manual construction of the reviewed event bulletin from the automatic bulletin. Largely due to the computational limitations of the 1990s when the systems were designed, the automatic parts of the process generally perform only a single pass over the waveform data. The waveform data are reanalyzed only when the manual review is performed. The greatest strain is placed on the system in the event of very large earthquakes followed by extensive aftershock sequences. The rapidly unfolding sequences of phase arrivals at the stations can present significant challenges to automatic procedures for phase association and event hypothesis creation. One such event happened on April 25, 2015, when an earthquake of magnitude 7.8 struck Nepal and was followed by several hundreds of aftershocks which were recorded globally. Figure 2 displays the event hypotheses in the fully automatic SL3 bulletin of the IDC in the first six days of this aftershock sequence, together with the final analystreviewed solutions for the same time interval. Panels (a) and (b) of Figure 2 are the real-life equivalents of panels (c) and (d) in Figure 1 . We see at a glance the problem faced by automatic algorithms in constructing an accurate representation of the sequence of seismic events.
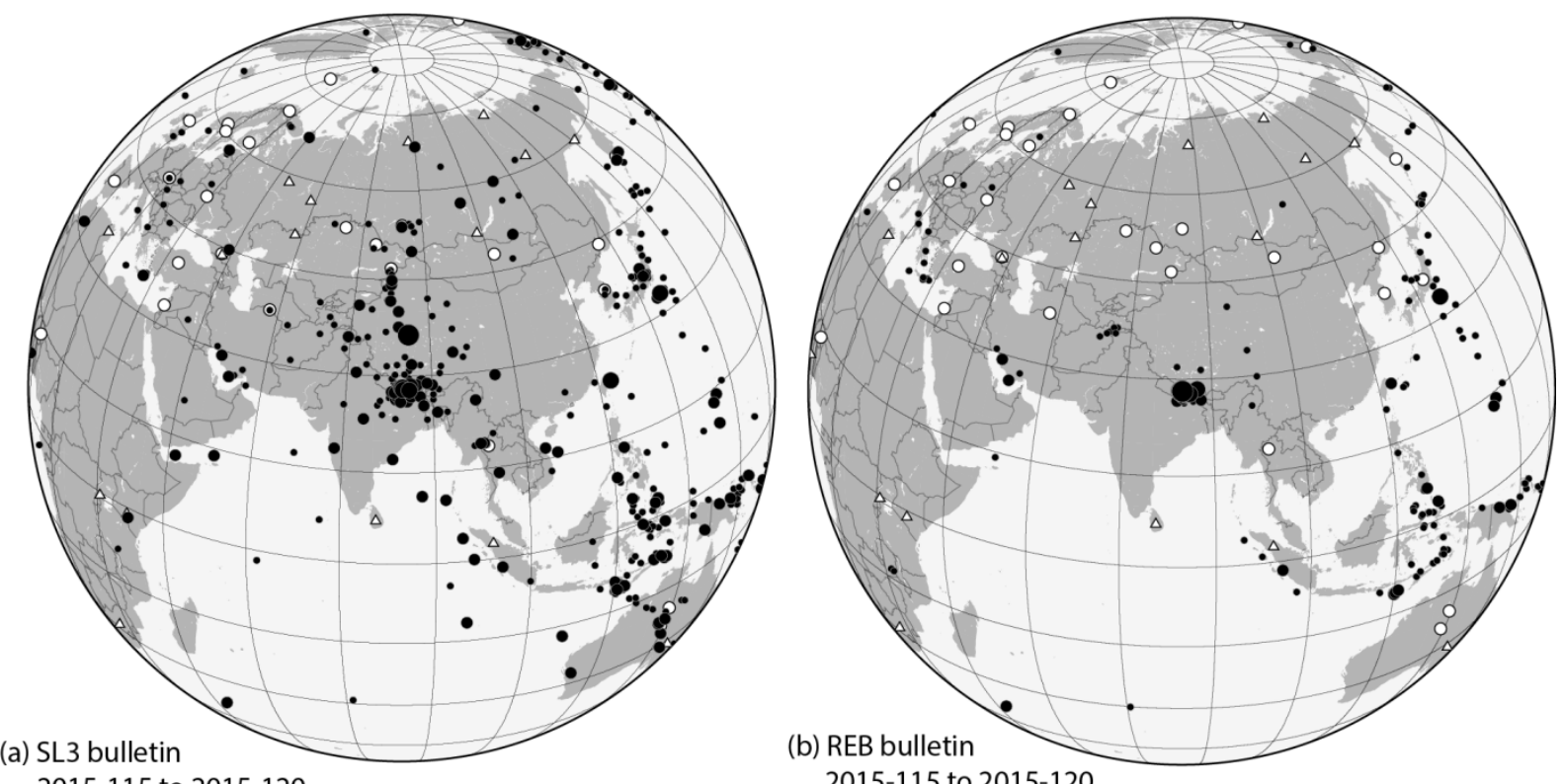

2015-115 to 2015-120 2015-115 to 2015-120 
Figure 2 Event location estimates from the fully automatic SL3 bulletin from the International Data Center (IDC) in Vienna for April 25 to April 30, 2015, and (b) event locations from the analystreviewed Reviewed Event Bulletin (REB) for the same time period.

In the aftershock sequence scenario, we have issues such as the overlap of signals (where first arrivals at key stations may be buried in the coda of signals from earlier events). A succession of events may be so rapid that qualitatively incorrect solutions with spuriously associated arrivals will often score more highly in automatic evaluations than the often incomplete sets of detections that correspond to the true origin time and source location. This is what leads to a scatter of preliminary event location estimates over continental distance scales (Figure 2 a). It is the enormous human effort involved in producing the result displayed in Figure 2 b) from the starting point displayed in Figure 2 a) that we seek to assist. There may be many strategies for improving the association algorithms, e.g. evaluating detection probabilities for different phases (Kværna and Ringdal, 2013) or a Bayesian reformulation of the problem (Arora et al., 2013). However, we wish to explore contextbased iterative solutions where new passes over the raw waveform data are performed using techniques which are optimized for the current state of seismicity.

We assume a situation where a large earthquake has occurred with many aftershocks anticipated in a given region, and aim to extract as much information as possible about the evolving sequence. The region of anticipated aftershock activity is likely to be determined by knowledge of the regional tectonics. Given a set of well-constrained events, we could then strip the parametric phase lists of detections associated with these aftershocks and therefore reduce the likelihood of false associations. Figure 3 illustrates how we may attempt to construct a timeline for event hypotheses within the aftershock region using some form of signal processing optimized for that particular source region on the available stations (panel a). It is not specified in the figure which algorithm we employ for this purpose - we could be using correlators or subspace detectors (e.g. Harris and Dodge, 2011; Barrett and Beroza, 2014) or some form of grid search method (see Lomax et al., 2014). 
With an accurate list of hypocenters and origin times, we may predict with considerable accuracy the sequence of phases anticipated at the different stations and these times can be used to mask out the corresponding phases (detected or not) from the parametric data streams (panel b). The reduced parametric data streams can then be sent again to the phase association process (c.f. Figure $1 \mathrm{~b}$ and c). While we may not guarantee a more accurate association of the remaining detections, we are likely to have reduced significantly the number of false event hypotheses involving arrivals from significant events from the aftershock region. A new association run based on the cleaned-up lists of parametric arrival data will result in a new set of event hypotheses, and the validity of these can be evaluated based upon the likelihood of detection, or non-detection, of phases depending upon the source location, magnitude and station detectability history (Kværna and Ringdal, 2013). In principle, the iteration between the event definition process and the association of seismic arrivals can continue until stopping criteria determine that no further information can be gleaned from the raw data and/or parametric data streams.

In this paper we provide a proof-of-concept demonstration that an offline (semi-)autonomous procedure could classify a certain category of events robustly, and with high accuracy, and remove all trace of them from the parametric data streams, increasing the likelihood that more general phase association algorithms will succeed on the remaining detections. We here focus on the aftershock sequence scenario although the concept is equally applicable to, for example, repeating mining explosions. An evaluation of a complete processing pipeline is beyond the scope of this paper. It is more our aim to make the case that, with today's increased computational resources, iterative techniques could be applied to the pipeline processes to relieve some of the human effort required for generating the final event bulletins. 
a)
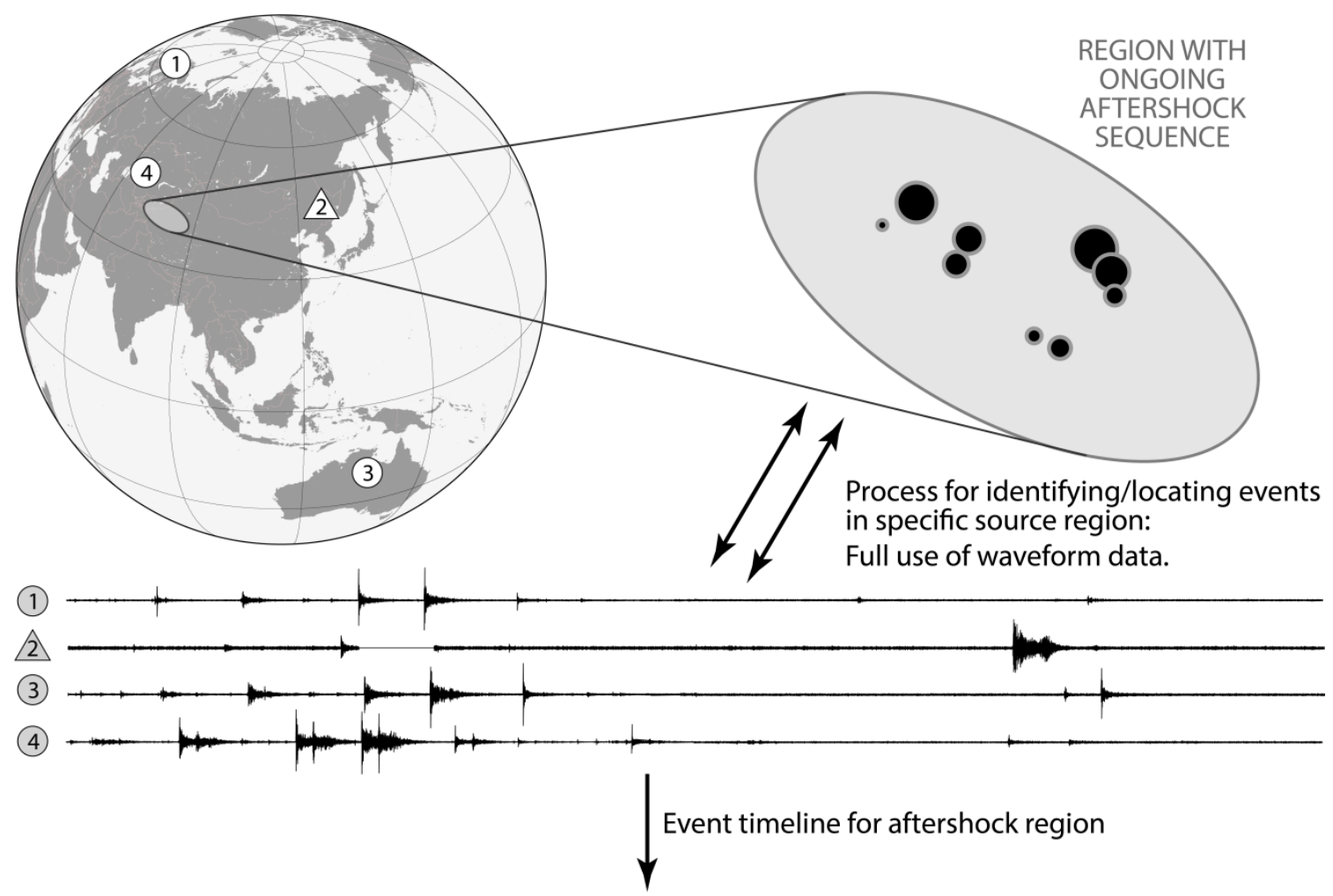

b)

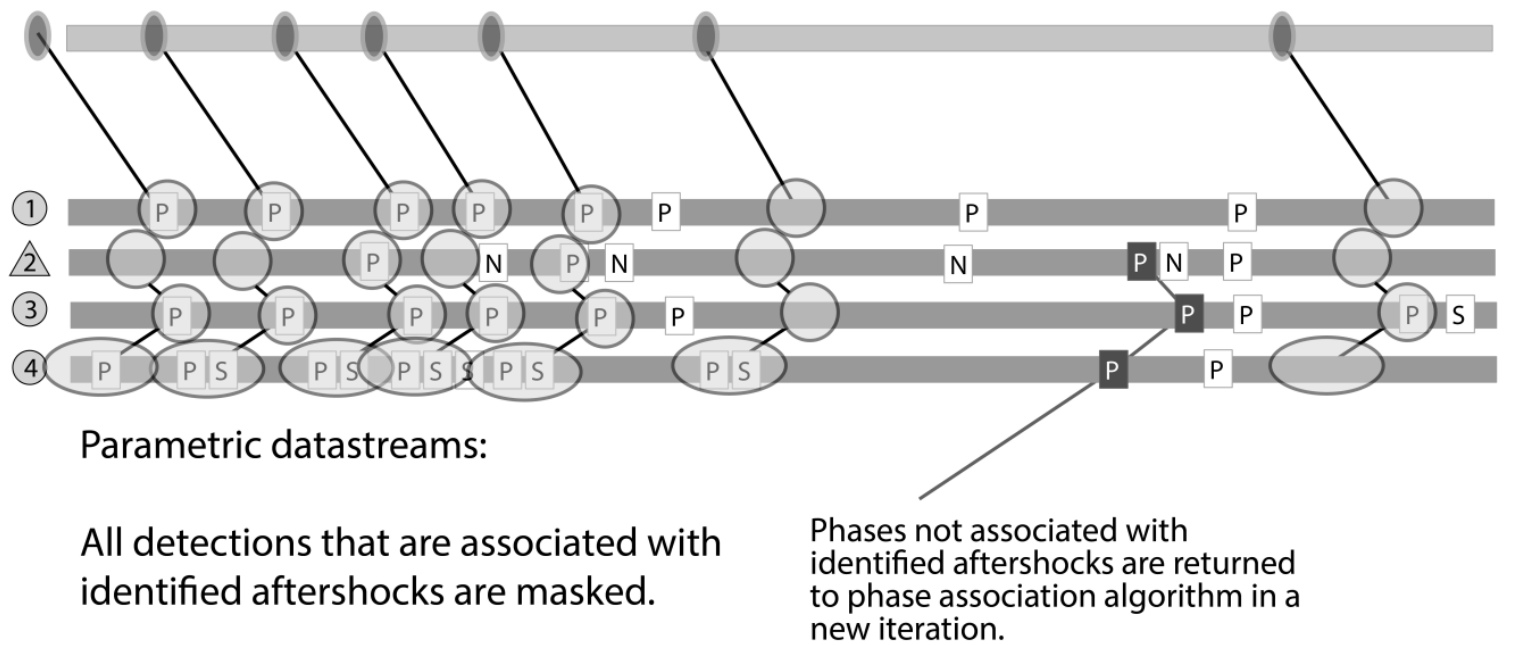

Figure 3 The processing scenario under the hypothesis of a developing aftershock sequence in a

limited geographical region, in which an offline process is initiated to detect and locate aftershocks accurately. The detection parameters associated with well characterized events (a) can be removed from the parametric data stream provided to the phase association algorithm (b). 


\section{STRATEGIES FOR CHARACTERIZING REPEATING SOURCES AND AFTERSHOCKS}

The workhorses for characterizing repeating seismicity, both from anthropogenic and natural sources, are so-called pattern or empirical signal detectors. In aftershock sequences from moderately sized earthquakes (e.g. up to magnitude 6.5), correlation and subspace detectors may perform exceptionally well in characterizing events with relatively limited numbers of templates covering the entire source region effectively (e.g. Gibbons and Ringdal, 2006; Harris and Dodge, 2011; Tang et al., 2014; Junek et al., 2015; Dodge and Harris, 2016). For larger events, such as the $M=7.8$ Nepal earthquake, the source regions are many times larger with dimensions up to and exceeding $100 \mathrm{~km}$. The waveform similarity between neighboring events decreases both with differences in source mechanism and with increasing hypocentral distance. It has been demonstrated for several large aftershock sequences that correlation methods are relatively ineffective at characterizing the seismicity, with only a very small proportion of events detected at distant stations showing significant signal similarity to other events (Slinkard et al., 2013).

When it becomes clear that an aftershock sequence is underway, we should be able to evaluate rapidly from historical performance which stations are likely to provide optimal detection capability for the given source region (e.g. Kværna and Ringdal, 2013). For array stations, we can choose a frequency band and a set of time delays that will optimize the likelihood of detecting a phase from that source region. The lowermost trace in Figure 4 shows a beam for the Warramunga array (WRA) in Australia steered with parameters which maximize the SNR for teleseismic P arrivals from the source region of the Mw 7.6 October 8, 2005, Kashmir earthquake (Mandal et al., 2007). WRA is a highly sensitive seismic array, far from cultural noise, and with excellent coherence for teleseismic signals. For each seismic phase (i.e. station and phase combination, e.g. teleseismic P at WRA) which is expected to be observed well from an event in our source region, we seek a characteristic function (CF). This is to say a time-series with a significant local maximum close to every occurrence of an arrival of this phase from an event in our sequence, and ideally with no significant local maxima at any other times. 
The auto-regressive Akaike Information Criterion (AR-AIC) procedure is commonly used for refining the onset time of a seismic arrival (see e.g. Leonard and Kennett, 1999, and references therein). In the procedure, an auto-regressive (AR) model is generated for the background noise prior to the detection time and a residual trace is formed containing, for each sample, the difference between the recorded value and the linear prediction from the preceding samples using this AR-model (e.g. Kay and Marple, 1981). The corresponding AIC trace attains the greatest (negative) value at the sample which best separates the part of the time-series well described by the noise AR-model and the part of the time-series not well described by this model. This estimate is frequently a very good approximation of the arrival time (e.g. Leonard, 2000) with the significance of the AIC minimum increasing with increasing contrast in the amplitude and spectral content between the signal and noise. In the absence of a signal onset hypothesis, we can simply perform this procedure on consecutive overlapping segments of the seismogram, calculating short AIC traces at regular intervals (e.g. at every sample or every other sample). Examples of such traces are displayed above the seismogram in Figure 4. Those windows which include a clear signal onset produce an AIC trace with a significant minimum close to the arrival time whereas windows in "pure noise" or "pure signal" do not. A summation of these overlapping AIC traces suppresses much of the random variability of the AIC functions in the absence of clear onsets and adds the significant minima constructively. The detection statistic resulting from multiplying this stack by -1 (Figure 4 , top trace) attains clear local maxima exceptionally close to the manually chosen onset time. This CF avoids the difficulty in determining signal onsets from STA/LTA traces and provides an alternative to procedures such as Kurtosis (e.g. Baillard et al., 2014).

It may be the case that the CF attains a significant local maximum for unrelated signals, given that it is only sensitive to an increase in amplitude of the incoming waveforms. For a seismic array, we may be able to screen and remove local maxima that are unrelated to our sequence by performing $f-k$ analysis or calculating the corresponding F-statistic (e.g. Selby, 2008). For a 3-component station, inconsistency with the anticipated particle motion (e.g. Vidale, 1986) may provide enough evidence 
to screen out a spurious local maximum. For a station at teleseismic distance, only a single P-phase is likely to be observed with a high SNR for each event. Later phases like PP and PcP are sometimes clear, but are usually of lower frequency and with considerably lower SNR. Stations at regional distances on the other hand may display a sequence of clear P- and S-type phases for each event. Grigoli et al. (2013) prescribe a system for automatic event detection and location with a regional network of 3-component stations using normalized CFs derived from Principal Component Analysis, whereby different CFs respond preferentially to either $\mathrm{P}$ or $\mathrm{S}$ phases, limiting the contribution to apparent S-phase energy resulting from P-arrivals and vice versa.

In Figure 5 we see waveforms, optimized for $\mathrm{P}$ and $\mathrm{S}$ phases as indicated, for three stations at regional distances from the Kashmir aftershock sequence. For most of the arrivals in Figure 5, both $\mathrm{P}$ and $\mathrm{S}$ are visible on both vertical and transverse horizontal rotations - both on the waveforms and on the corresponding CFs. For each station, at the time of a given local CF-maximum, there are likely to be local maxima on both P and S-optimized CFs. If the CF with the highest value at the time of the local maximum is identified, as indicated in Figure 5, a good strategy is simply to attribute that trigger to the targeted phase and to set all other CFs for that station to zero at that time. If the station is an array, we will probably be in addition able to differentiate P- and S- phases using $f-k$ analysis. A station which results in multiple triggers which are not easily differentiated using simple signal processing algorithms is probably best excluded from the network. However, it is worth bearing in mind that the purpose of this work is to characterize well events from a targeted region that are well recorded over an extensive network. Any one trigger will only define an event if numerous additional triggers, detected on CFs for other stations, are consistent with the arrivals predicted from a single location and a common origin time. Consequently, the system is likely to be able to cope with a significant number of triggers from unrelated sources. 


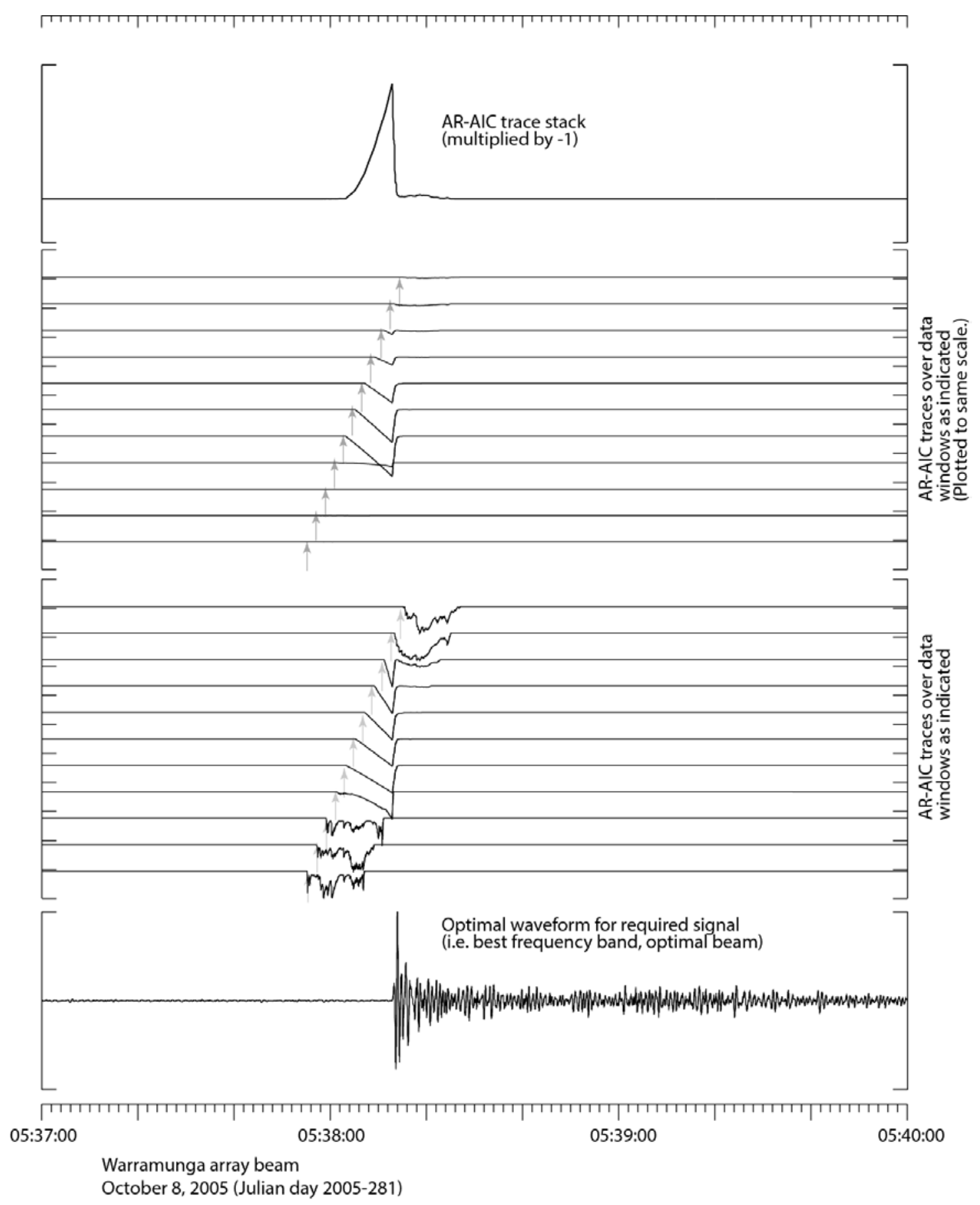

Figure 4 Generation of a trace for arrival detection formed by continuous stacking of AR-AIC functions calculated in overlapping data segments. The beam uses backazimuth 322 degrees, apparent velocity $22 \mathrm{~km} / \mathrm{s}$ and the frequency band 1-5 Hz. For each time window used for calculating an AR-AIC trace, the window length has total duration 15 seconds, and the AR noise model is calculated using the data from only the first 5 seconds of the current window. The AR linear prediction filter uses 8 coefficients. 
(a)

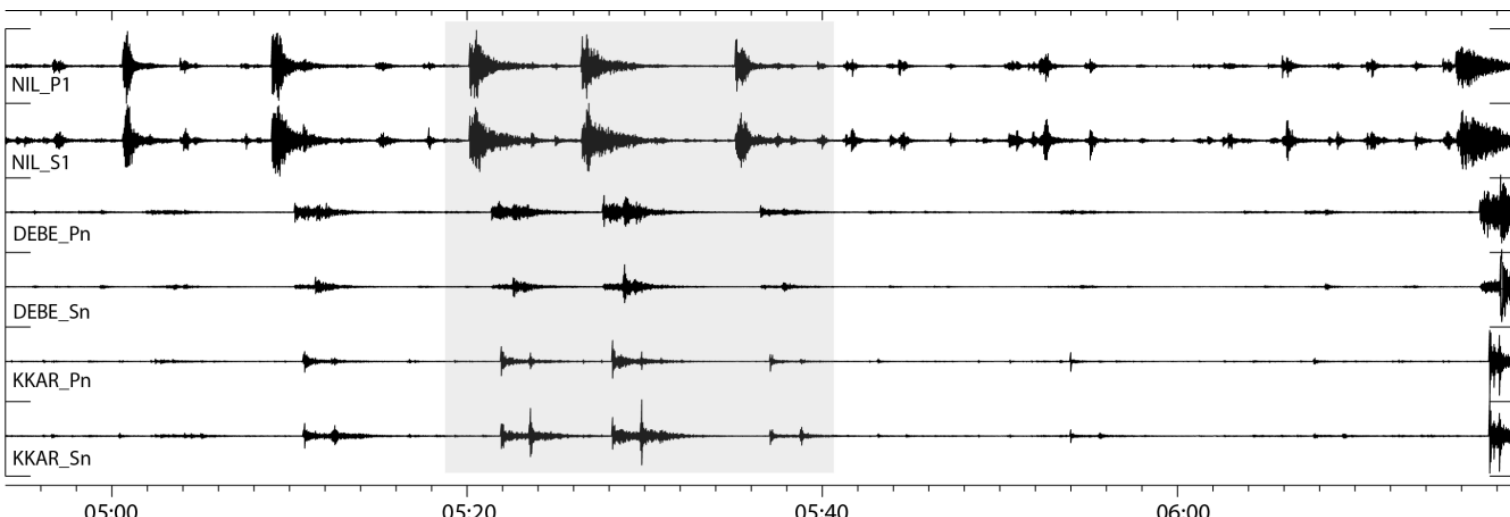

(b)

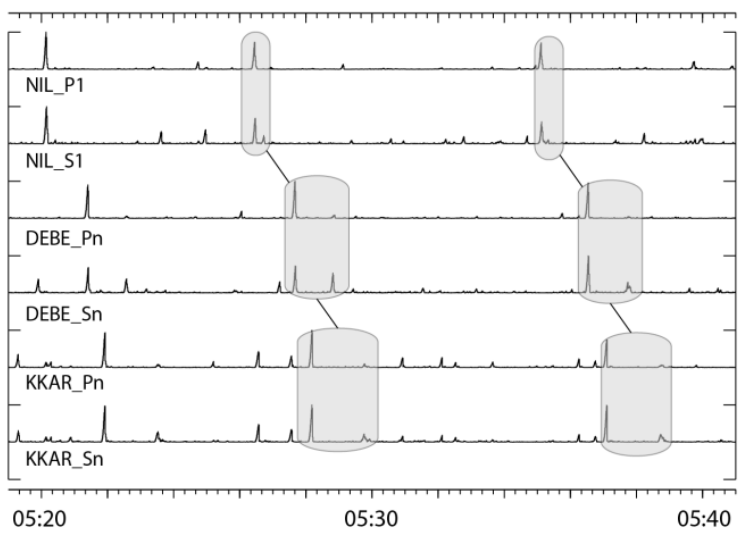

Time (UTC) 2005-281 (October 8)

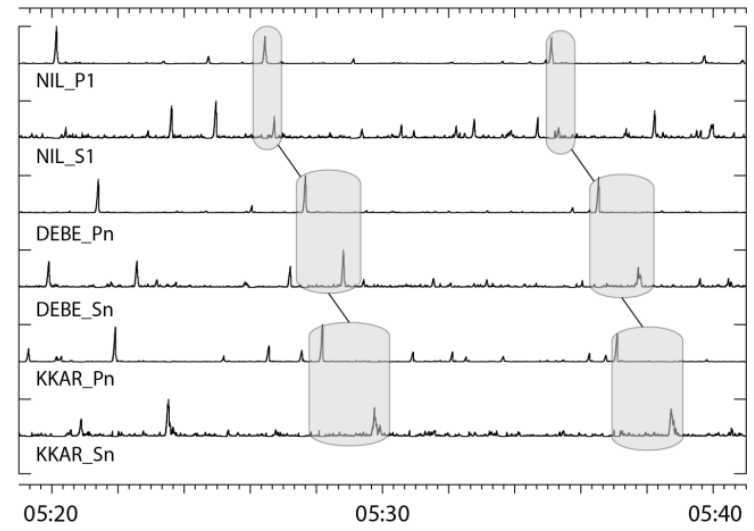

Figure 5 Traces from 3 stations at regional distances (between 1 and 9 degrees) from the October

8, 2005, Kashmir earthquake optimized for the detection of $P$ and $S$ waves as indicated (a). Panel

(b) shows the corresponding AR-AIC traces for the highlighted time interval in panel (a) and panel

(c) shows the same traces but where, for each station, for any significant local maximum on either

P- or S-optimized traces, the trace with the lowest value is set to zero in a $\mathbf{1 0}$ second window

centered on this local maximum. 
There are many grid-search possibilities for generating event hypotheses (see e.g. Kao and Shan, 2004; Grigoli et al., 2013; Lomax et al., 2014). However, the defining feature of the AR-AIC characteristic functions displayed in figures 4 and 5 is the accuracy of the arrival time estimates. This property means that with well-constrained traveltimes, for a given phase arrival and hypocenter hypothesis (i.e. latitude, longitude and depth), the origin time of the hypothetical event is constrained to a very short time-window. Dealing with global observations, the traveltimes of Pwaves are becoming exceptionally well characterized (e.g. Simmons et al., 2012) and we can typically predict teleseismic P-waves with an uncertainty significantly less than one second (Myers et al., 2015). Our strategy is to form a 2-D or 3-D grid of candidate hypocenters in the aftershock region and, for every trigger detected on the set of CFs, attempt to form an event. For each grid point, the defining trigger time will define a single event hypothesis with an origin time uniquely determined by the trigger time and traveltime. We then project out from this origin hypothesis to all of the other stations using the corresponding traveltimes and count the number of local maxima that fall within a short time window of the predicted arrival time. For grid points far from the true hypocenter, it is very likely that no or few other CF local maxima will be reached and these event hypotheses can be discarded immediately. The problem of event definition then becomes one of identifying the best of the event hypotheses which are consistent with local maxima on the CFs for many different phases. This will be described in the next section for a case study using the first few days of the April 25, 2015, Gorkha aftershock sequence, Nepal. 


\section{CASE STUDY: THE 2015 GORKHA AFTERSHOCK SEQUENCE, NEPAL}

The Mw 7.8 Gorkha, Nepal, earthquake of April 25, 2015, was recorded with a high SNR at many IMS seismic arrays at teleseismic distances. The top trace in Figure 6a) shows a beam that is deemed optimal for the detection of teleseismic P from Nepal aftershocks for the ARCES array in Norway. The amplitudes of the signals from the few largest events dominate the trace, although no fewer than 142 signal detections occur in the time-window indicated which provided a slowness estimate consistent with a Nepal P-signal. In the lower trace of Figure 6a) is the corresponding AR-AIC CF, calculated as displayed in Figure 4). The AR-AIC function has a smaller dynamic range than the waveforms themselves and the signal onsets from the smaller events are more visible. Similar optimal beams and corresponding AR-AIC characteristic functions were calculated for a number of other IMS seismic arrays (ASAR, BVAR, CMAR, ESDC, FINES, GEYT, ILAR, KSRS, KURK, MKAR, NOA, SONM, TORD, WRA, YKA, and ZALV) and 3-component stations (AKTO, ARU, KIRV, NRIK, TIXI, and YAK). The distribution of these stations relative to the source region is displayed in Figure 7 and it is clear that all of these stations are at far-regional or teleseismic distances. Only P-phases were sought for this study. 

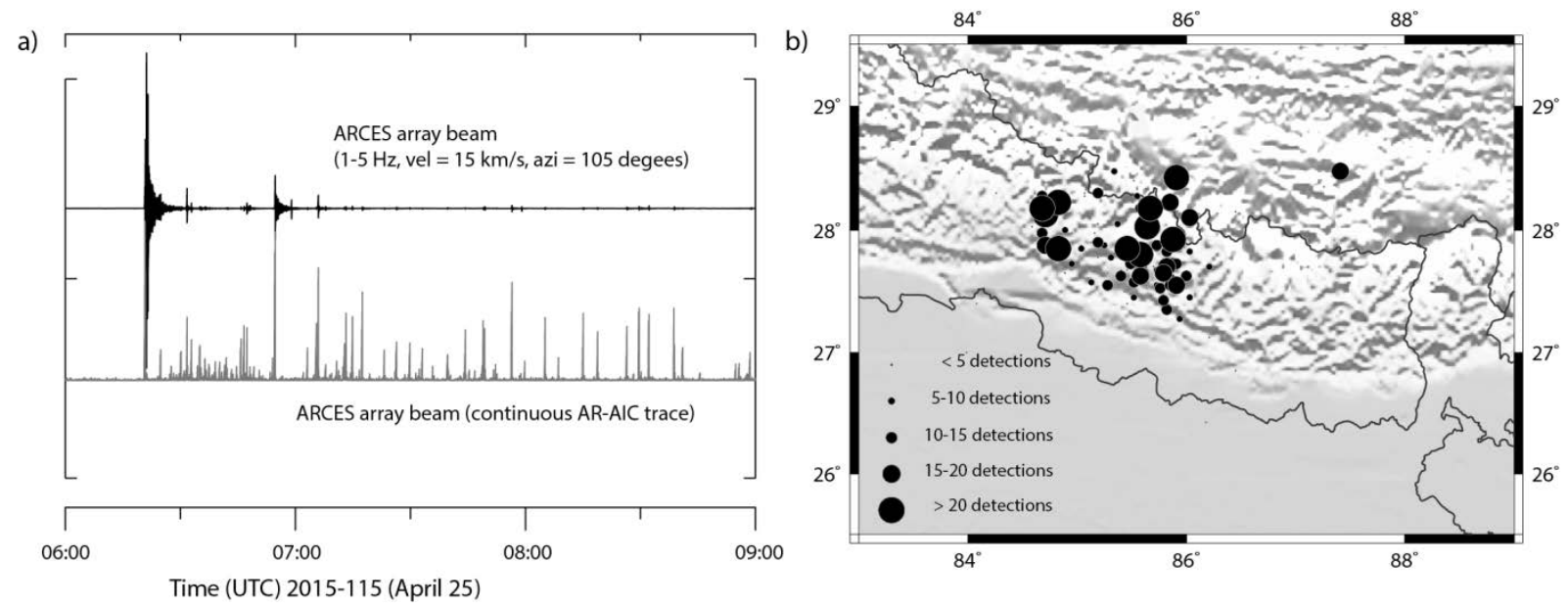

Figure 6. Optimal beam on the ARCES seismic array for detecting teleseismic $P$-arrivals from the Nepal region and the corresponding continuous AR-AIC trace (a). Fully automatic event hypotheses from a grid search scan of the source region scaled according to the number of AR-AIC detections associated with each event hypothesis for April 25 and April 262015 (b). 158 events are displayed in panel (b) compared with 294 events in the REB in the same time period.

To judge a sensible threshold for determining a trigger on the AR-AIC CF requires inspection of the traces; a nominal threshold of 50 appeared to pass those local maxima which clearly were associated with a required arrival. It should be noted that, in this case study, only the value of the CF was considered; all associated parametric information, such as that provided by $\mathrm{f}-\mathrm{k}$ analysis, was ignored. With the CF functions for each array calculated, these time-series were searched for events within one-hour-long time-intervals. A 2-dimensional grid of trial hypocenters was defined between 26 and 29 degrees north, 83 and 39 degrees east, and with a fixed depth of $15 \mathrm{~km}$. With 121 grid points in latitude and 201 grid points in longitude we have approximately $3 \mathrm{~km}$ spacing laterally for hypocenter candidates. This grid definition was based upon calculations of aftershock locations carried out by Adhikari et al. (2015) and Dixit et al. (2015), and traveltimes for each phase were calculated a priori for every node in the grid using the LLNL-G3D 3-dimensional P-wave model of Simmons et al. (2012). 
Each local maximum in the set of CFs exceeding the threshold was considered to be a trigger and a grid search was performed for each such trigger. Every node in the grid was used to form an event hypothesis with the origin time first obtained by subtracting the pre-calculated traveltime from the trigger time. A set of predictions was then calculated for arrivals on every other CF using the corresponding traveltimes. If, and only if, an AR-AIC local maximum greater than the nominal threshold fell within a predefined time tolerance (set to 1.1 seconds in this study) of the predicted arrival time would this phase be associated with the origin hypothesis. Any origin hypothesis with fewer than a predefined number of supporting phases was immediately rejected. All event hypotheses with more than this number (here set to 4 ) were stored, together with the number $N_{i}$ of associated phases and a 1-norm measure of the time residual vector. All parameters were chosen empirically and are subject to continual evaluation. The time tolerance for example might have to be increased if the onset time estimates or the traveltime calculations were to indicate that this were necessary. Given that this procedure is carried out for each trigger on the AR-AIC characteristic functions, a very high number of event hypotheses can be formed - many essentially identical, but differing slightly in the origin time and associated time residual. On the other hand, the calculation involves only the addition and subtraction of pre-calculated traveltimes and evaluating whether or not the CF exceeds a threshold close to the predicted arrival time. This makes the procedure far more rapid than an algorithm that involves, for example, stack-and-delay of different CFs. With all event hypotheses evaluated for the hour-long segment, the maximum number $N_{\max }$ of associated phases was found and the winning event hypothesis was selected as the hypothesis with $N_{\max }$ associated phases with the minimum residual vector. This best hypothesis was saved and all local maxima within 10 seconds of each arrival time predicted from this origin were set to zero. This search was performed again with the now reduced set of CF local maxima and repeated until no qualifying event hypotheses remained, with the associated phases being removed for each event accepted. 
This grid search procedure described here is clearly a brute-force method but, given the reliability of the arrival time estimates and the high speed at which each candidate hypocenter can be evaluated, a significant geographical region can be searched for preliminary event definitions. The method is not global and necessarily targets only a limited source region. (We are using a limited number of specially optimized detection statistics on a limited number of stations.) All passed event hypotheses occurring at nodes close to the boundary of the grid are deemed most likely to have originated outside of the search window and are deleted. We cannot exclude the possibility of triggers from events outside of the search region but the minimum number of phases needed to define an event hypothesis, and the exclusion of events occurring close to the boundary, appears to have kept this case study free of such examples. If the selection criteria were to require additional parameters, such as backazimuth, the likelihood of including foreign events would decrease further. The event hypotheses automatically determined from the set of AR-AIC characteristic functions formed for April 25 and 26 are displayed in Figure $6 \mathrm{~b}$ ). The size of the symbols relates only to the number of phases for which a local maximum above the threshold value could be associated, using the traveltime alone, with the candidate hypocenter. The spatial distribution of these event hypotheses is very similar to that obtained from manual relocations (e.g. Hayes et al., 2015). 


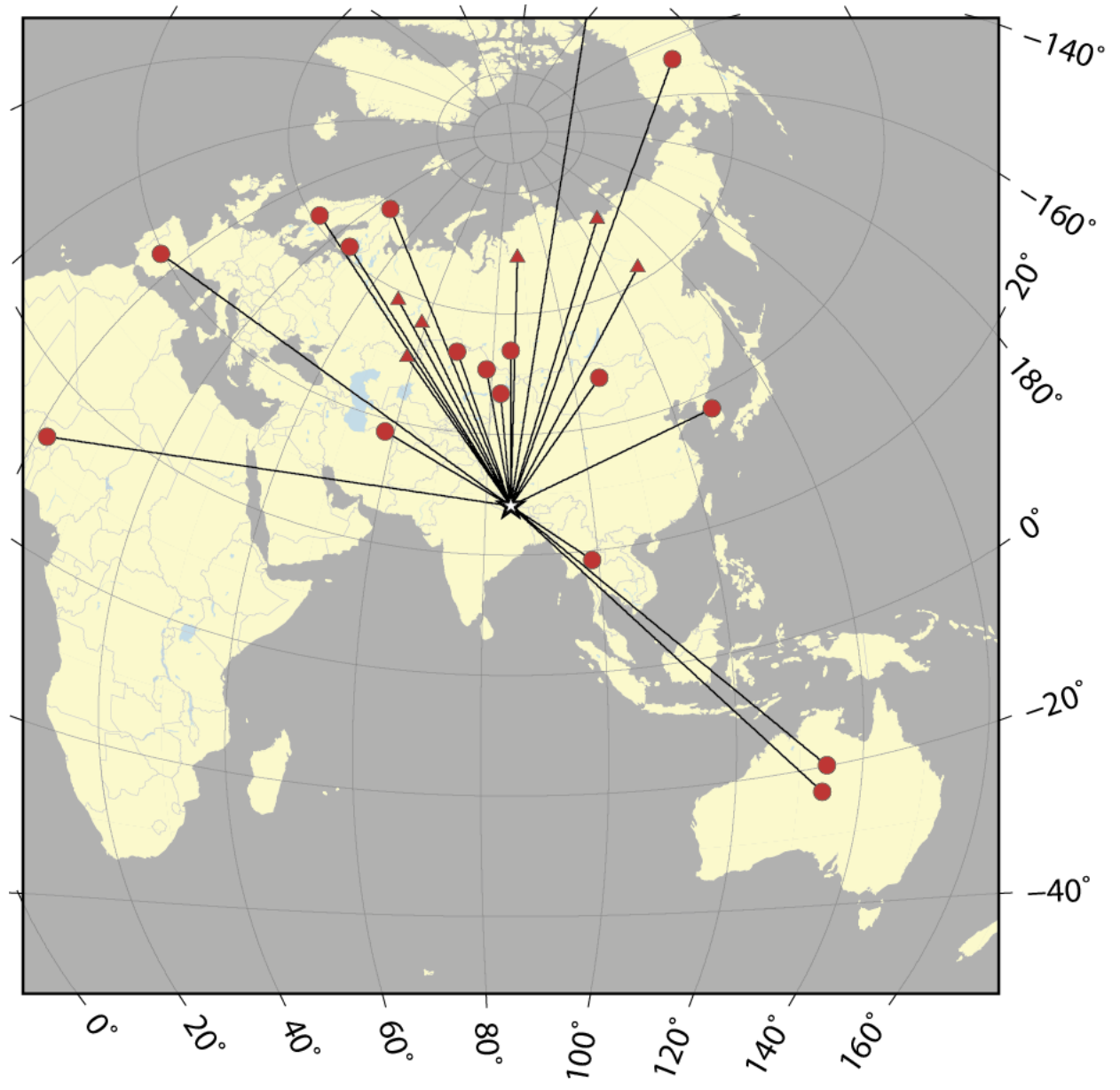

Figure 7. IMS arrays (circles) and 3-component stations (triangles) used to form characteristic functions for optimized detection of aftershocks of the Nepal earthquake. 

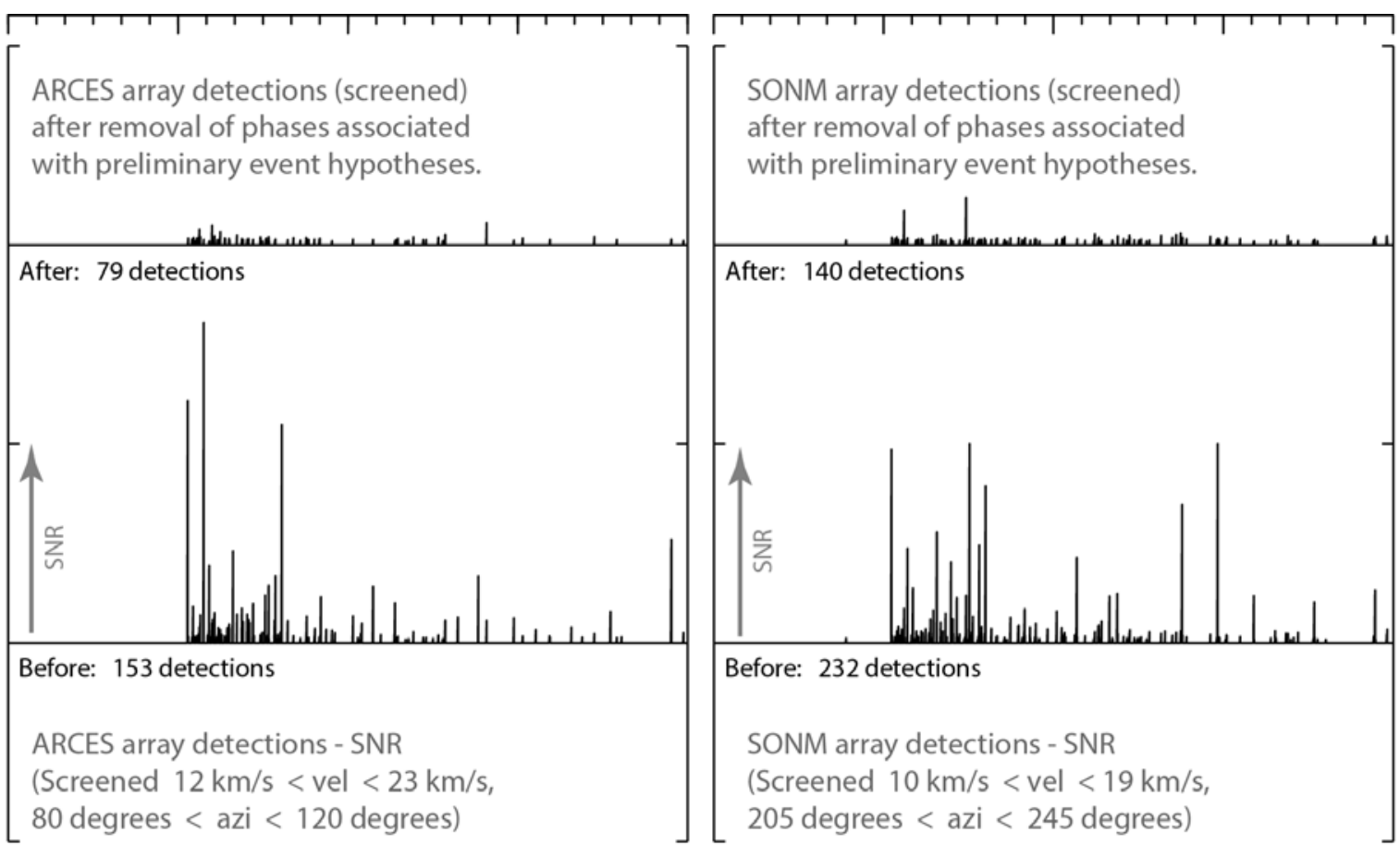

Before: 232 detections

SONM array detections - SNR

(Screened $10 \mathrm{~km} / \mathrm{s}<$ vel $<19 \mathrm{~km} / \mathrm{s}$,

205 degrees < azi < 245 degrees)

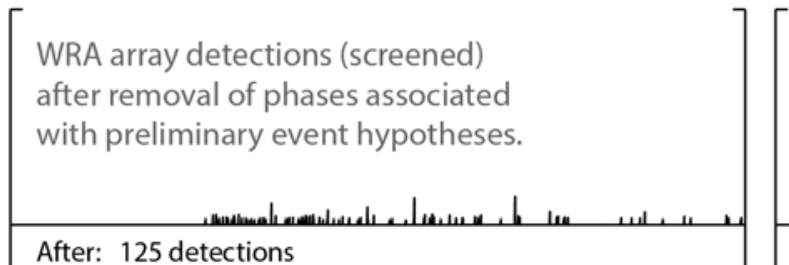

ZALV array detections (screened)

after removal of phases associated

with preliminary event hypotheses.

After: 113 detections

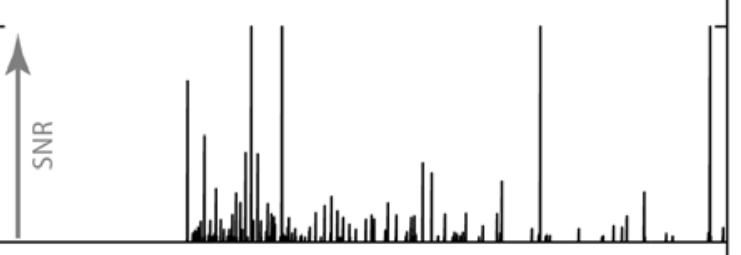

Before: 215 detections

WRA array detections - SNR

(Screened $15 \mathrm{~km} / \mathrm{s}<$ vel $<20 \mathrm{~km} / \mathrm{s}$,

310 degrees < azi < 335 degrees)
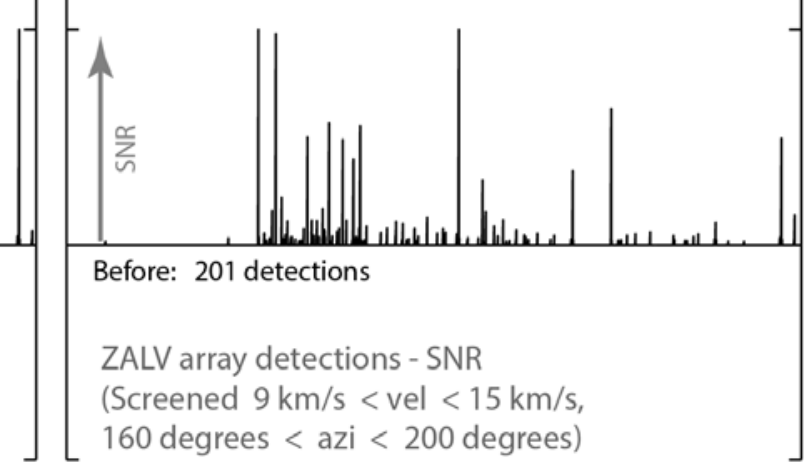

Before: 201 detections

ZALV array detections - SNR

(Screened $9 \mathrm{~km} / \mathrm{s}<$ vel $<15 \mathrm{~km} / \mathrm{s}$

160 degrees < azi < 200 degrees)

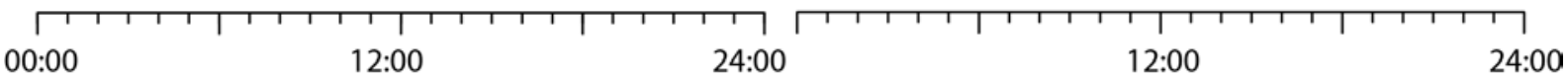

Time (UTC) 2015-115 (April 25)

Figure 8. A comparison of detection lists on 4 IMS array stations before and after screening from

the preliminary set of event hypocenters displayed in Figure 6. Each vertical bar represents a

detection that falls within the range of expected parameters with height proportional to SNR. The

heights of the arrows indicate an SNR of 200 at which a few of the strongest signals have been

clipped artificially for display purposes. 
The events displayed in Figure 6b) constitute a surprisingly satisfactory preliminary event bulletin for the aftershock region; the median distance between the fully automatic hypocenter estimates and the final REB solutions is $23.7 \mathrm{~km}$. There are fewer automatic event hypocenters than are present in the REB for this time period, due to the strict criteria for defining events on the AR-AIC characteristic functions (only arrivals with a significant SNR generate local maxima of the CFs which exceed the nominal threshold). It should be noted that many REB events are defined only by signals in the coda of previous events and are constructed from phases with very low SNR. Almost all such events are only detected by virtue of the coherence detected on seismic arrays. We recall however that our goal is not to form a complete catalog, but rather construct the most accurate possible description of the best defined events in the aftershock region such that all associated phases can be stripped from the parametric detection lists (c.f. Figure 3b).

In Figure 8 we see the effect of removing all phases associated with this set of events from the detection lists for four primary IMS seismic arrays. Slightly fewer than half of the detections for each station have been eliminated from the detection lists, and only detections with lower SNR remain. It should also be noted that many of the remaining detections are likely to be coda detections; the correct identification and removal of these is likely to improve the results of subsequent phase association algorithms. Of the automatic event hypotheses in the SL3 bulletin that were deemed to be of high quality, falling within 15 seconds and $150 \mathrm{~km}$ of REB events, the median distance between automatic and reviewed hypocenters is $23.5 \mathrm{~km}$ (very similar to that for the grid search hypocenters found here). The key to reducing the workload on analysts is both an improvement of the quality of valid event hypotheses and a reduction of the number of spurious event hypotheses. Our aim is to point to methods for achieving both. 


\section{CONCLUSIONS}

We have highlighted the difficulties that extensive aftershock sequences following very large earthquakes present for agencies attempting to generate near real-time earthquake catalogs. It is self-evident that an increase in the number of events requiring classification will generate an additional burden to the human resources required. However, the increase in the required effort usually increases disproportionately given that the rapid sequence of arrivals from the vast number of aftershocks often confounds the automatic phase association algorithms, resulting in poorer quality automatic event bulletins. Although progress is being made in improving the systems which form the automatic event hypotheses, we propose an iterative strategy whereby phases and signals associated with the aftershocks are removed from the parametric datastreams by a separate subprocess.

The optimal process for classifying the aftershocks will depend upon the nature of the sequence. For smaller earthquakes, with aftershocks limited to a relatively small geographical region, pattern detectors (e.g. correlators, subspace detectors and matched field detectors) may characterize the entire source region quite efficiently. For larger events (e.g. above magnitude 7) there is often little waveform similarity within the usable frequency bands for the widely spaced aftershocks. For this reason, we advocate the use of more general procedures for aftershock classification (possibly in combination with the use of pattern detectors). We present a case study of the Mw 7.8 May 25, 2015, Nepal earthquake for which we attempt to detect and locate aftershocks in an offline process. This process comprises three parts:

1. Generation of special targeted optimal datastreams from multiple stations. For seismic arrays, this involves forming optimal beams.

2. Construction of a characteristic function (CF) for each such datastream. We implement a continuous AR-AIC trace which produces local maxima very close to the signal onset times.

3. A grid search of the aftershock source region using the CFs for the different stations. 
With a provisional event list generated, we remove all associated phases from the incoming datastreams and run the phase association algorithm on the screened detection lists. Even with a very basic offline procedure for the Nepal sequence, we removed almost half of the detections associated with the aftershock sequence. The proportion of detections screened would almost certainly increase if more sophisticated methods were employed to identify those phases associated with the aftershocks (e.g. Harris and Kværna, 2010). Significant process is being made with empirical signal detectors at scales from local (e.g. Wang et al., 2015) to regional (e.g. Yoon et al., 2015), and global (e.g. Dodge and Walter, 2015) and it is conceivable that multiple processes could run simultaneously to try to characterize the ongoing sequence. Of greatest importance is the idea that the detection and location pipeline no longer has to be serial. A contextual, iterative, scheme may perform multiple passes over the waveform data to build an increasingly accurate picture of the current seismicity at all scales. 


\section{DATA AND RESOURCES}

Website of the Pacific Northwest Seismic Network: https://pnsn.org/about (last accessed January 2016).

Website of the National Earthquake Information Center:

http://earthquake.usgs.gov/contactus/golden/neic.php (last accessed January 2016).

The text of the Comprehensive Nuclear-Test-Ban Treaty is found at https://www.ctbto.org/the-

$\underline{\text { treaty/treaty-text/ (last accessed January 2016). }}$

The LLNL-G3D 3-dimensional P-wave velocity model is available from

https://missions.llnl.gov/nonproliferation/nuclear-explosion-monitoring/global-3d-seismic-

tomography together with software for performing the traveltime calculations (last accessed January 2016).

\section{ACKNOWLEDGEMENTS}

This work was supported in part by the Air Force Research Laboratory under contract number FA9453-13-C-0270 and in part by the Norwegian Ministry of Foreign Affairs. It was also performed in part under the auspices of the U.S. Department of Energy by Lawrence Livermore National Laboratory under contract DE-AC52-07NA27344. Lawrence Livermore National Security, LLC. This is LLNL Contribution LLNL-JRNL-683501-DRAFT.

All maps generated by the GMT package, Wessel and Smith (1995). 


\section{REFERENCES}

Adhikari, L. B., Gautam, U. P., Koirala, B. P., Bhattarai, M., Kandel, T., Gupta, R. M., Timsina, C., Maharjan, N., Maharjan, K., Dahal, T., Hoste-Colomer, R., Cano, Y., Dandine, M., Guilhem, A., Merrer, S., Roudil, P., Bollinger, L. (2015), The aftershock sequence of the 2015 April 25 Gorkha-Nepal earthquake, Geophysical Journal International, 203, 2119-2124, doi:10.1093/gji/ggv412

Arora, N. S., Russell, S., and Sudderth, E. (2013), NET-VISA: Network Processing Vertically Integrated Seismic Analysis, Bulletin of the Seismological Society of America, 103, 709-729, doi:10.1785/0120120107

Bache, T. C., Bratt, S. R., Swanger, H. J., Beall, G. W. and Dashiell, F. K. (1993), Knowledge-based interpretation of seismic data in the Intelligent Monitoring System, Bulletin of the Seismological Society of America, 83, 1507-1526

Baillard, C., Crawford, W. C., Ballu, V., Hibert, C., and Mangeney, A. (2014), An Automatic KurtosisBased P- and S-Phase Picker Designed for Local Seismic Networks, Bulletin of the Seismological Society of America, 104, 394-409, doi:10.1785/0120120347

Barrett, S. A. and Beroza, G. C. (2014), An Empirical Approach to Subspace Detection, Seismological Research Letters, 85, 594-600, doi:10.1785/0220130152

Dahlman, O., S. Mykkeltveit, H. Haak (2009), Nuclear Test Ban: Converting Political Visions to Reality, Springer Science+Business Media B.V, Dordrecht, The Netherlands ISBN: 978-1-4020-6883-6 (Print) 978-1-4020-6885-0 (Online) 10.1007/978-1-4020-6885-0

Dahlman, O., J. Mackby, S. Mykkeltveit, H. Haak (2011), Detect and Deter: Can Countries Verify the Nuclear Test Ban? Springer Science+Business Media B.V, Dordrecht, The Netherlands. ISBN: 978-94-007-1675-9 (Print) 978-94-007-1676-6 (Online) doi:10.1007/978-94-007-1676-6 
Dixit, A. M., Ringler, A. T., Sumy, D. F., Cochran, E. S., Hough, S. E., Martin, S. S., Gibbons, S., Luetgert, J. H., John Galetzka, Surya N. Shrestha, Sudhir Rajaure, Daniel E. McNamara (2015), StrongMotion Observations of the M 7.8 Gorkha, Nepal, Earthquake Sequence and Development of the N-SHAKE Strong-Motion Network, Seismological Research Letters, 86, 1533-1539, doi:10.1785/0220150146

Dodge, D. A. and Harris, D. B. (2016), Large-Scale Test of Dynamic Correlation Processors: Implications for Correlation-Based Seismic Pipelines, Bulletin of the Seismological Society of America, (early online at present), doi:10.1785/0120150254.

Dodge, D. A. and Walter, W. R. (2015), Initial Global Seismic Cross-Correlation Results: Implications for Empirical Signal Detectors, Bulletin of the Seismological Society of America, 15, 240-256, doi:10.1785/0120140166

Douglas, A. (2013), Forensic Seismology and Nuclear Test Bans, Cambridge University Press, Cambridge, ISBN 9781107033948

Gibbons, S. J. and Ringdal, F. (2006), The detection of low magnitude seismic events using arraybased waveform correlation, Geophysical Journal International, 165, 149-166, doi:10.1111/i.1365-246x.2006.02865.x

Grigoli, F., Cesca, S., Amoroso, O., Emolo, A., Zollo, A., and Dahm, T. (2014), Automated seismic event location by waveform coherence analysis, Geophysical Journal International, 196 1742-1753, doi:10.1093/gii/ggt477

Harris, D. B. and Dodge, D. A. (2011) “An Autonomous System for Grouping Events in a Developing Aftershock Sequence" Bulletin of the Seismological Society of America, 101, 763-774, doi:10.1785/0120100103 
Harris, D. B. and T. Kværna (2010), Superresolution with seismic arrays using empirical matched field processing, Geophysical Journal International, 182, 1455-1477, doi:10.1111/i.1365$\underline{246 x .2010 .04684 . x}$

Hayes, G. P., Briggs, R. W., Barnhart, W. D., Yeck, W. L., McNamara, D. E., Wald, D. J., Nealy, J. L., Benz, H. M., Gold, R. D., Jaiswal, K. S., Marano, K., Earle, P. S., Hearne, M. G., Smoczyk, G. M., Wald, L. A., and Samsonov, S. V. (2015), Rapid Characterization of the 2015 Mw 7.8 Gorkha, Nepal, Earthquake Sequence and Its Seismotectonic Context, Seismological Research Letters, 86, 1557-1567, doi:10.1785/0220150145

Junek, W. N., Kværna, T., Pirli, M., Schweitzer, J., Harris, D. B., Dodge, D. A., and Woods, M. T. (2015), Inferring Aftershock Sequence Properties and Tectonic Structure Using Empirical Signal Detectors, Pure and Applied Geophysics, 172, 359-373, doi:10.1007/s00024-014-0938-0

Kao, H. and Shan, S. (2004), The Source-Scanning Algorithm: mapping the distribution of seismic sources in time and space, Geophysical Journal International, 157, 589-594, doi:10.1111/j.1365-246x.2004.02276.x

Kay, S. M. and Marple, S. L. (1981), Spectrum analysis-A modern perspective, Proceedings of the IEEE, 69, 1380-1419, doi:10.1109/proc.1981.12184

Kennett, B. L. N., Engdahl, E. R., and Buland R. (1995). Constraints on seismic velocities in the Earth from travel times, Geophysical Journal International, 122, 108-124, doi:10.1111/j.1365246x.1995.tb03540.x

Kværna, T. and F. Ringdal (2013), Detection Capability of the Seismic Network of the International Monitoring System for the Comprehensive Nuclear-Test-Ban Treaty, Bulletin of the Seismological Society of America, 103, 759-772, doi:10.1785/0120120248 
Le Bras, R., Swanger, H., Sereno, T., Beall, G., Jenkins, R., Nagy, W. and Henson, A. (1994), Global Association: Final Report, Scientific Applications International Corporation Technical Report SA $94 / 1155$

Leonard, M. (2000), Comparison of Manual and Automatic Onset Time Picking, Bulletin of the Seismological Society of America, 90, 1384-1390, doi:10.1785/0120000026

Leonard, M., and B. L. N. Kennett (1999), Multi-component autoregressive techniques for the analysis of seismograms, Physics of the Earth and Planetary Interiors, 113, 247-263, doi:10.1016/s0031-9201(99)00054-0

Lomax, A., Michelini, A., and Curtis, A. (2014), Earthquake Location, Direct, Global-Search Methods, In Encyclopedia of Complexity and Systems Science, editor Robert A. Myers, 1-33, Springer, New York, $10.1007 / 978-3-642-27737-5 \quad 150-2$

Mandal, P., Chadha, R. K., Kumar, N., Raju, I. P., and Satyamurty, C. (2007), Source Parameters of the Deadly Mw 7.6 Kashmir Earthquake of 8 October, 2005, Pure and Applied Geophysics, 164, 1963-1983, doi:10.1007/s00024-007-0258-8

Myers, S. C., Simmons, N. A., Johannesson, G., and Matzel, E. (2015), Improved Regional and Teleseismic P-Wave Travel-Time Prediction and Event Location Using a Global 3D Velocity Model, Bulletin of the Seismological Society of America, 105, 1642-1660, doi:10.1785/0120140272

Ringdal, F. and Kværna, T. (1989), A multi-channel processing approach to real time network detection, phase association, and threshold monitoring, Bulletin of the Seismological Society of America, 79, 1927-1940

Selby, N. (2008), Application of a Generalized F Detector at a Seismometer Array, Bulletin of the Seismological Society of America, 98, 2469-2481, doi:10.1785/0120070282 
Simmons, N. A., Myers, S. C., Johannesson, G. and Matzel, E. (2012), LLNL-G3Dv3: Global P wave tomography model for improved regional and teleseismic travel time prediction, Journal of Geophysical Research, 117, B10302, doi:10.1029/2012jb009525

Slinkard, M. E., Carr, D. B., Young, C. J. (2013), Applying Waveform Correlation to Three Aftershock Sequences, Bulletin of the Seismological Society of America, 103, 675-693, doi:10.1785/0120120058

Tang, C., Lin, C. and Peng, Z. (2014), Spatial-temporal evolution of early aftershocks following the 2010 ML 6.4 Jiashian earthquake in southern Taiwan, Geophysical Journal International, 199, 1772-1783, doi:10.1093/gii/ggu361

Wang, J., Templeton, D. C. and Harris, D. B. (2015), Discovering new events beyond the catalogueapplication of empirical matched field processing to Salton Sea geothermal field seismicity, Geophysical Journal International, 203, 22-32, doi:10.1093/gii/ggv260

Wessel, P. and W. H. F. Smith (1995), New version of the generic mapping tools released, EOS Transactions, American Geophysical Union 76, no. 33, 329, doi:10.1029/98eo00426.

Yoon, C. E., O'Reilly, O., Bergen, K. J., and Beroza, G. C. (2015), Earthquake detection through computationally efficient similarity search, Science Advances, 1, e1501057-e1501057, doi:10.1126/sciadv.1501057 